SHORT REPORT

\title{
Copper deficiency myeloneuropathy and pancytopenia secondary to overuse of zinc supplementation
}

\section{J Rowin, S L Lewis}

J Neurol Neurosurg Psychiatry 2005;76:750-751. doi: 10.1136/jnnp.2004.046987

The haematological complications of acquired copper deficiency have been well documented, but the neurological complications have only recently been reported. An illustrative case of copper deficiency myeloneuropathy with pancytopenia is presented and the potential aetiologies and neurological manifestations of this deficiency state discussed.

$\mathrm{T}$ he haematological complications of acquired copper deficiency have been well documented. ${ }^{1-3}$ Dietary copper deficiency causes spinal cord dysfunction and ataxia in ruminants, known as swayback disease. A human form of "swayback disease" has recently been described. ${ }^{4-10}$ Early recognition and treatment of this newly recognised deficiency state may reverse or prevent its serious complications. We present an illustrative case of copper deficiency myeloneuropathy with pancytopenia and discuss the potential aetiologies and neurological manifestations of this deficiency state.

\section{CASE REPORT}

A 53 year old woman presented with progressive gait imbalance. Four months before presentation she began to develop tingling and numbness in the fingers and feet. One month later she visited an outside emergency room for evaluation of back pain. She was found to have a normocytic anaemia with a haemoglobin concentration of $8.3 \mathrm{~g} / \mathrm{dl}$ (normal 11.7 to $15.7 \mathrm{~g} / \mathrm{dl}$ ) and a white blood cell count of $3.2 \times 10^{3}$ (normal 3.8 to $10.8 \times 10^{3}$ ). She received two units of packed red blood cells, bringing her haemoglobin to $11.5 \mathrm{~g} / \mathrm{dl}$. Iron supplementation was prescribed for presumed iron deficiency anaemia. Three days after visiting the emergency room she awoke with increased numbness in the extremities and she began using a cane because of unsteadiness.

On presentation her neurological examination showed increased tone and mild weakness in the legs. Muscle stretch reflexes were hyperactive at the knees and normal elsewhere, with absent Babinski signs. Vibratory and proprioceptive sensation was decreased in the feet. She had mild dysmetria in the upper extremities. Her gait was wide based, spastic, and ataxic. Her drug treatment included citalopram hydrobromide and lorazepam for depression and anxiety, and supplemental iron and zinc. She self initiated zinc supplementation a year or more before presentation as treatment for recurrent sinusitis.

Previous work up included magnetic resonance imaging (MRI) of the brain and entire spine, an EMG, and a lumbar puncture. The brain and spinal MRI investigations were unremarkable. Nerve conduction studies showed a mild length dependent motor and sensory polyneuropathy involving the lower extremities. Although there were demyelinating features, particularly in the peroneal motor conduction, the criteria for a primarily demyelinating polyneuropathy were not met. Specifically, there was absent sural sensory response, borderline low amplitude peroneal motor response (distal $2.0 \mathrm{mV}$ (normal >2.2); proximal $1.7 \mathrm{mV}$ (normal $>2.2)$ ), prolongation of the peroneal distal motor latency $(8.3 \mathrm{~ms}$ (normal $<5.7 \mathrm{~ms})$ ), and slowing of motor conduction velocities (peroneal, $23.2 \mathrm{~m} / \mathrm{s}$ (normal >38.0)); tibial, $33.5 \mathrm{~m} / \mathrm{s}$ $($ normal $>41)$ ). The tibial distal latency and amplitude and the median sensory and median and ulnar motor conductions were normal. Cerebrospinal fluid was acellular with a normal protein and a normal banding pattern.

Laboratories at presentation included a white blood cell count of $2.6 \times 10^{3}$ with a mean corpuscular volume of 112.5 $\mu \mathrm{M}$ (normal 80 to 100). The electrolytes, liver enzymes, glucose, thyroid stimulating hormone, fatty acid profile, vitamin B-12, folic acid, homocysteine, methylmalonic acid, vitamin E, serum protein immunoelectrophoresis, serum protein immunofixation, angiotensin converting enzyme, free fatty acids, and arylsulphatase A were all normal. Antinuclear antibody, SS-A and SS-B antibodies, antigliadin antibody, and antiphospholipid antibodies were negative. Syphilis, Lyme, and HIV serology were negative.

The serum copper level was markedly low at $7 \mu \mathrm{g} / \mathrm{dl}$ (normal 70 to 155), as was the serum caeruloplasmin, at 2.1 $\mathrm{mg} / \mathrm{dl}$ (normal 22.9 to 43.1 ). Serum zinc was raised at 2.28 $\mu \mathrm{g} / \mathrm{ml}$ (normal 0.66 to 1.10 ). The patient's zinc supplement was inspected. Each capsule contained $50 \mathrm{mg}$ of elemental zinc in the form of zinc gluconate. The recommended dosage, according to the label, was one capsule daily $(333 \%$ of the recommended daily dose of $15 \mathrm{mg}$ ). The patient described taking four to eight capsules daily; she would increase the dose according to the severity of her symptoms, ingesting up to $400 \mathrm{mg} /$ day.

A diagnosis of zinc induced copper deficiency myeloneuropathy associated with pancytopenia was made. The zinc was discontinued. She received one dose of intravenous copper chloride (2.0 mg elemental copper) and was subsequently started on oral copper sulphate supplementation $(2.0 \mathrm{mg} /$ day elemental copper). A bone marrow biopsy taken 12 days after the start of copper supplementation was normocellular with slightly left shifted maturation in all cell lines. No tumour cells were present. Her copper level, white blood cell count, and mean corpuscular volume normalised after three months of treatment. There was significant but incomplete improvement in her gait and spasticity at six months. There was also some improvement in her nerve conduction studies, with the appearance of a normal sural sensory response (latency 4.0 ms (normal <4.2), amplitude $6.5 \mu \mathrm{V}$ (normal >5.0)). There was no improvement in the motor conduction times.

\section{DISCUSSION}

We believe that our patient developed copper deficiency myeloneuropathy and pancytopenia secondary to overuse of zinc supplements, as in a case reported by Kumar et al..$^{5}$ Our patient was ingesting approximately 20 times the recommended daily allowance of zinc. Both copper and zinc are absorbed in the stomach and proximal duodenum. Excess 
zinc levels cause an upregulation of metallothionein production in the enterocytes. Copper has a higher affinity for metallothionein than zinc, so it displaces zinc from metallothionein. Copper then remains in the enterocytes and is sloughed off into the intestinal tract and eliminated. ${ }^{1}$

Other aetiologies of copper deficiency myeloneuropathy have been reported, including five cases secondary to gastric resection..$^{4}{ }^{9}{ }^{10}$ Other cases have an unclear aetiology with no known external sources of zinc, but with high plasma zinc concentrations. $^{71011}$ Copper deficiency with haematological manifestations has been reported in malnutrition, prematurity, and parenteral or enteral feeding that does not include copper. $^{3}$

There are some features in common between the neurological manifestations of copper deficiency and multiple sclerosis. Interestingly, the copper chelating agent cuprizone is used as a neurotoxicant in a mouse model of CNS demyelination. ${ }^{12}$ Although our patient did not show central demyelinating lesions on MRI, Prodan and Holland reported CNS white matter lesions in the brains of their patients with copper deficiency. ${ }^{13}$ Also, hyperintensity on T2 weighted MR images can be seen in the dorsal spinal cord of some patients with copper deficiency myelopathy. ${ }^{4}{ }^{9} 10$

Gregg et al $^{3}$ reported a case of copper deficiency with severe neutropenia and anaemia. The patient also reportedly had an associated severe optic neuropathy and peripheral neuropathy. Clioquinol, a copper zinc chelating antibiotic, ${ }^{14}$ caused subacute myelo-optico-neuropathy in nearly 10000 patients in Japan before the banning of its use in 1970. ${ }^{15}$ This raises the question of whether acquired optic neuropathy, such as seen in optic myeloneuropathy of $\mathrm{Cuba}^{16}$ or other presumed nutritional/toxic optic myeloneuropathies, ${ }^{17}$ may be associated with copper deficiency.

Gastric bypass surgery for obesity is associated with neuropathy as well as other neurological complications. It appears that approximately $40 \%$ of the cases of post-gastric reduction surgery neuropathy are associated with thiamin or vitamin B-12 deficiency, but in the remaining $60 \%$ no vitamin deficiency is found. ${ }^{18}$ As there are reports of copper deficiency myeloneuropathy after gastric bypass surgery for various indications in the absence of parenteral or enteral nutrition, ${ }^{469}$ copper deficiency should be considered in cases of neuropathy after weight reduction surgery.

As with vitamin B-12 deficiency, the neurological manifestations of copper deficiency may be seen with or without the haematological manifestations, and with or without abnormalities on MRI imaging of the brain and spinal cord. ${ }^{9}$ The associated anaemia of copper deficiency may be macrocytic, as in our case, microcytic, or normocytic. ${ }^{4}$ Therefore a high index of suspicion is necessary in patients at risk. Copper therapy $(2 \mathrm{mg} / \mathrm{d})$ generally leads to an early recovery of the haematological abnormalities, followed by variable recovery of the neurological symptoms.

Copper deficiency should be considered in the differential diagnosis of multiple sclerosis, subacute combined degeneration of the cord, optic myeloneuropathy, post-gastric reduction surgery neuropathy, or in other cases of myelopathy, optic neuropathy, or polyneuropathy where nutritional deficiency or overuse of zinc supplementation is suspected. Prompt recognition and treatment may improve the prognosis for neurological recovery.

\section{Authors' affiliations}

J Rowin, University of Illinois at Chicago, Department of Neurology and Rehabilitation, Section of Neuromuscular Disease, Chicago, Illinois, USA S L Lewis, Rush University Medical Center, Department of Neurological Sciences, Chicago

Competing interests: none declared

Correspondence to: Dr Julie Rowin, University of Illinois at Chicago, Department of Neurology and Rehabilitation, Section of Neuromuscular Disease, Chicago, IL 60612, USA; rowin@uic.edu

Received 14 June 2004

Revised version received 23 August 2004

Accepted 25 August 2004

\section{REFERENCES}

1 Fiske DN, McCoy HE, Kitchens CS. Zinc-induced sideroblastic anemia: report of a case, review of the literature, and description of the hematologic syndrome. Am J Hematol 1994;46:147-50.

2 Simon SR, Branda RF, Tindle BH, et al. Copper deficiency and sideroblastic anemia associated with zinc ingestion. Am J Hematol 1988;28:181-3.

3 Gregg XT, Reddy V, Prchal JT. Copper deficiency masquerading as myelodysplastic syndrome. Blood 2002;100:1493-5.

4 Schleper B, Stuerenburg HJ. Copper deficiency-associated myelopathy in a 46-year-old woman. J Neurol $2001 ; 248: 705-6$.

5 Kumar N, Gross JB, Ahlskog JE. Myelopathy due to copper deficiency. Neurology 2003;61:273-4.

6 Kumar N, McEvoy KM, Ahlskog JE. Myelopathy due to copper deficiency following gastrointestinal surgery. Arch Neurol 2003;60:1782-5.

7 Hedera P. Myelopolyneuropathy and pancytopenia due to copper deficiency and high zinc levels of unknown origin: further support for existence of a new zinc overload syndrome. Arch Neurol 2003;60:1303-6.

8 Prodan $\mathrm{Cl}$, Holland NR, Wisom PJ, et al. Myelopathy due to copper deficiency. Neurology 2004;62:1655-6.

9 Kumar N, Crum B, Petersen RC, et al. Copper deficiency myelopathy. Arch Neurol 2004;61:762-6.

10 Kumar N, Gross JB, Ahlskog JE. Copper deficiency myelopathy produces a clinical picture like subacute combined degeneration. Neurology 2004;63:33-9.

11 Prodan C, Holland NR, Wisdom PJ, et al. CNS demyelination associated with copper deficiency and hyperzincemia. Neurology 2002;59:1453-6.

12 Matsushima GK, Morell P. The neurotoxicant, cuprizone, as a model to study demyelination and remyelination in the central nervous system. Brain Pathol 2001;11:107-16.

13 Prodan Cl, Holland NR. CNS demyelination from zinc toxicity? Neurology 2000;54:1705-6.

14 Rosenberg RN. Metal chelation therapy for Alzheimer disease. Arch Neurol 2003;60:1678.

15 Konagaya $M$, et al. Clinical analysis of longstanding subacute myelo-opticoneuropathy: sequelae of clioquinol at 32 years after its ban. J Neurol Sci 2004;218:85-90

16 Carelli V, Ross-Cisneros FN, Sadun AA. Optic nerve degeneration and mitochondrial dysfunction: genetic and acquired optic neuropathies. Neurochem Int 2002:40:573-84.

17 Oluwole OSA, Onabolu AO, Link $\mathrm{H}$, et al. Persistence of tropical ataxic neuropathy in a Nigerian community. J Neurol Neurosurg Psychiatry 2000;69:96-101

18 Chang CG, Adams-Huet B, Provost DA. Acute post-gastric reduction surgery (APGARS). Neuropathy Obes Surg 2004;14:182-9. 